\title{
Vigorous Activity Usage of Self-Identified Leaders: A Descriptive Quantitative Analysis
}

\author{
Joseph Williams \\ Misssouri State University, Springfield, USA \\ Email: josephwilliams@missouristate.edu
}

How to cite this paper: Williams, J. (2020). Vigorous Activity Usage of Self-Identified Leaders: A Descriptive Quantitative Analysis. Open Journal of Leadership, 9, 53-69. https://doi.org/10.4236/oj1.2020.91004

Received: June 26, 2019

Accepted: February 16, 2020

Published: February 19, 2020

Copyright $\odot 2020$ by author(s) and Scientific Research Publishing Inc. This work is licensed under the Creative Commons Attribution International License (CC BY 4.0).

http://creativecommons.org/licenses/by/4.0/ (c) (i) Open Access

\begin{abstract}
The aim of the study was to conduct original research to better understand the relationship between participation in vigorous physical activity and the frequency in use of five servant leadership characteristics. The relationship between vigorous physical activity participation and self-reported servant leadership characteristics was evaluated in 162 women and men with a median age of $36 \pm 9$ years. Participants' self-reported vigorous physical activity participation was evaluated with the International Physical Activity Questionnaire (IPAQ), and self-reported servant leadership characteristics were evaluated using the Servant Leadership Questionnaire (SLQ). Vigorous physical activity participation was not statistically related to self-reported servant leadership characteristics. Participants in this study on average participated in approximately 43 minutes of vigorous physical activity each day of the week. These participants had much higher vigorous physical activity participation rates than the national average. Participants' perception of their servant leadership characteristics was also high. Findings suggest that the exclusive use of servant leadership philosophy to promote vigorous physical activity participation is not needed, leaving open the possibility that any type of leadership approach could be used to promote physical activity participation.
\end{abstract}

\section{Keywords}

Servant Leadership, Leadership, Vigorous Activity, Metabolic Syndrome

\section{Introduction}

Metabolic syndrome and the declining health across America are impacting productivity and employee welfare (Boles, Pelletier, \& Lynch, 2004). Evidence suggests organizations that build cultures of health by emphasizing employee well-being have competitive advantages in the marketplace and yield greater 
workforce value (Thayer et al., 2013). Research has shown vigorous activity measurably improves personal health and the prevention of obesity, elevated blood sugar, and negative lipid profiles (Swain \& Franklin, 2006; Williams, 2004). However, less than $5 \%$ of the U.S. population participate in vigorous activity (swimming, running, or strength training) on a regular basis (Tudor-Locke, Johnson, \& Katzmarzyk, 2010).

Participation in physical activity can positively impact personal health, mood, overall wellness, and, to a lesser extent, decision making (Collingwood, 1997; Hupin et al., 2015). Physical activity and athletic prowess have long been associated with desirable leadership characteristics (e.g., see myths about Greek and Roman gods), but few research has been done to systematically explore the association between physical activity and leadership. As a first step toward clarifying some aspects of the relationship between physical activity and leadership, a research project was conducted to collect empirical data to examine this relationship.

\section{Physical Activity}

With the burgeoning development of kinesiology starting in the 1970s, researchers began to investigate the interactions between physical activity and life skills (Wrynn, 2003). The literature, as a collective, suggests a positive association between physical activity and individual traits, including some literature on the relationship between certain leadership traits and physical activity (Beachamp, Welch, \& Hulley, 2007; McDowell-Larsen et al., 2002; Robson, 2013). However, the direct primary research showing associations between physical activity participation and leadership practices remains in its infancy (Beachamp et al., 2007; Himelhoch, 2014; Kwoh, 2013; McDowell-Larsen et al., 2002; Robson, 2013). Nonetheless, research showing the benefits of physical activity participation, including improvements in psychological factors, such as resilience and well-being, and sociological factors, such as social integration, cooperation, and respect, creates a compelling reason to continue to advance research into the promotion of physical activity participation (Bailey, 2006; Collingwood, 1997; Ferron, Narring, Cauderay, \& Michaud, 1999; Taylor, Sallis, \& Needle, 1985; Wankel \& Berger, 2018).

Regarding activity styles and approaches, vigorous modalities have recently caught the attention of the general public as an effective and concise training method to achieve physical and social psychological benefits (Ozanian, 2015; Collingwood, 1997). Generally, vigorous exercise includes activities like jogging, biking, and swimming laps. Recently, one version of vigorous activity called high-intensity interval training and heart rate monitor training, has become popularized by programs like Soul Cycle, Pure Barre, CrossFit, Orange Theory, or 9-Round. In these types of programs, intense, physically demanding, work periods range from 5 seconds to 8 minutes long and are usually performed at $80 \%$ 95\% of a person's maximum heart rate (Kravitz, 2014; Norton, Norton, \& Sad- 
grove, 2010). The work periods result in escalated breathing patterns and the inability to say more than a few words without taking a breath. As a health beneficial activity, current U.S. physical activity guidelines recommend accumulating at least 75 minutes each week of vigorous-intensity aerobic physical activity in bouts or episodes of at least 10 minutes per episode to receive the health benefits (Centers for Disease Control and Prevention, 2013). Individuals often will exercise at a vigorous level for short periods of time and then take moderate or light activity breaks between vigorous bouts to accumulate vigorous and moderate physical activity participation time, which helps to meet the guidelines.

This type of vigorous activity has been shown in recent studies to increase the effectiveness in aerobic fitness by conferring greater cardioprotective benefits, including improved systolic blood pressure, lipid profiles, and body fat percentages compared to moderate-intensity physical activity routines (Swain \& Franklin, 2006). In one study, regular vigorous physical activity participation was shown to increase the lifespan of adults aged 45 - 75 years by up to 13 years regardless of sex, body fat percentage, and the existence of pre-existing health conditions, such as cardiovascular disease and diabetes (Gebel et al., 2015). Vigorous activity has also been found to maximize health outcomes and the prevention of medical conditions such as stroke, hypertension, and colon and breast cancers, regardless of age (Karlsen, Aamot, Haykowsky, \& Rognmo, 2017).

In addition to the cardiovascular benefits, vigorous activity participation has also been linked to increased neural plasticity and brain health (Ahlskog, 2011). In the study by Ahlskog (2011), participation in midlife vigorous activity was related to a reduction in the later risk of Parkinson disease-caused dementia and the risk of mild cognitive impairment. Many other studies have related vigorous activity to a host of positive outcomes in relation to osteoporosis (Gutin \& Kasper, 1992), sleep (Youngstedt, 2005), body weight (Williams, 2004), and anxiety and depression (Morgan, 1985). Collectively, the research supports vigorous physical activity participation as a concise method of accumulating regular physical activity that is able to confer positive, long-term neurological and systemic health outcomes. Even as the benefits of vigorous activity are known and promoted, the challenge remains that less than $5 \%$ of the U.S. population participates in vigorous activity on a regular basis (Tudor-Locke, Johnson, \& Katzmarzyk, 2010).

\section{Leadership}

Beginning with "great-man" theories (Northouse, 2012), early studies into leadership began around the industrial revolution as there was increased awareness of the effect of differential leadership styles on work productivity. As the study of leadership has progressed, many different leadership perspectives have been identified (e.g., transformational leadership, leader-member exchange, adaptive leadership; Northouse, 2012). One leadership perspective, called servant leadership, has been useful in studying the characteristics, behaviors, and impacts of 
individuals who practice it (Keith, 2013). Servant leadership focuses on how the leader empowers and stimulates the members of the group in the pursuit of goals (Greenleaf, 1977). This leadership perspective starts with the belief that the way in which the leader helps the growth and development of the group will lead to positive outcomes and goal fulfillment (Greenleaf, 1977). Servant leadership suggests that group success is attained through legitimately empowering each group member by being a servant to the members' needs, including the need to be healthier, wiser, freer, more autonomous, and more likely themselves to become servant leaders (Greenleaf, 1977; Keith, 2013).

Servant leadership characteristics. In an attempt to create a reliable and functional measure for empirical research of servant leadership characteristics, Barbuto \& Wheeler (2006) distilled the common servant leadership characteristics into five factors. These factors attempt to capture the overall essence of servant leadership theory using conceptually and empirically distinct characteristics. The five factors include:

1) Altruistic calling. Altruistic calling, as defined by Barbuto \& Wheeler (2006), is a leader's inherent desire to make a positive difference in other people's lives. Altruistic calling encompasses generosity and a natural desire for a leader to serve other people's needs before their own (Barbuto \& Wheeler, 2006). An example item from the questionnaire is: I sacrifice my own interests to meet others' needs.

2) Emotional healing. Emotional healing, as defined by Barbuto \& Wheeler (2006), is a leader's utilization of empathy and listening to foster an environment where personal recovery from hardships, trauma, and failure are allowed and embraced. This characteristic also includes the wisdom to realize this process takes time, is individualized, and requires professional dialogue that is repercussion-free (Barbuto \& Gifford, 2010; Barbuto \& Wheeler, 2006). An example item from the questionnaire is: I am talented at helping others heal emotionally.

3) Wisdom. Wisdom, according to Barbuto \& Wheeler (2006) is defined as a combination of the leader's awareness of surroundings and the anticipation of consequences, providing an ideal balance between what is perfect and what is practical. This characteristic requires the leader to display high levels of knowledge and utility (Barbuto \& Wheeler, 2006). An example item from the questionnaire is: I am good at anticipating the consequences of decisions.

4) Persuasive mapping. Persuasive mapping is defined as a leader's encouragement of others to have a vision of the organizational future and persuasively give reasons to motivate others to work for common goals (Barbuto \& Wheeler, 2006). A leader with these skills uses efficient planning, goal visioning, and clear, yet motivating, communication (Barbuto \& Gifford, 2010). An example item from the questionnaire is: I offer compelling reasons to get others to do things.

5) Organizational stewardship. Organizational stewardship is an ethical responsibility of a leader and is defined as the leader's ability to guide his or her organization to make a positive impact on the community, delivered programs, 
and those who are exposed to the organizational processes and outcomes (Barbuto \& Wheeler, 2006). An example item from the questionnaire is: I am preparing the organization to make a positive difference in the future.

\section{Conceptual Link between Physical Activity and Servant Leadership}

The deliberate practice of both vigorous physical activity and servant leadership requires attitudes, skills, and beliefs that seem to have conceptual overlaps. For example, both vigorous physical activity participants and servant leadership practitioners share an attitude that strives to work outside one's comfort zone and strive to overcome challenges. Similarly, the set of skills required to regularly participate in vigorous physical activity and to consistently practice servant leadership characteristics includes discipline, strategic thinking, planning, and clear communication. Finally, conceptually the overlap between vigorous physical activity participation and servant leadership practice includes the beliefs that the promotion of health is the desired outcome. These three conceptual pivot points (attitudes, skills, and beliefs) between physical activity and servant leadership provide a foundation upon which to explore these variables. Therefore, the next step is to empirically explore the nature and strength of the relationship between these variables.

The purpose of this study was to investigate the relationship between vigorous physical activity participation and servant leadership characteristics. Using a descriptive, cross-sectional, correlation study, participants completed an online survey to assess their self-reported vigorous physical activity participation using the International Physical Activity Questionnaire (IPAQ) and self-reported servant leadership characteristics using the Servant Leadership Questionnaire (SLQ). This study sought to provide interested professionals with empirical information they can use to promote physical activity participation and positive leadership practices.

\section{Method}

\subsection{Participants}

Participants were recruited from a local gym population and from among the faculty of a southern Midwestern state university to participate in the online survey. Data were collected using the online survey platform Qualtrics (Qualtrics Version October 2017). Participant recruitment resulted in 217 individuals who started the survey. After cleaning the data, 162 responses were considered complete and were used in the remaining analysis (Figure 1). The average age of the 162 respondents was 37.00 years $(S D=9.23)$ with a median of 36.00 years. The lowest age was 21 years and the highest was 64 years. Of the 162 participants, there were 85 (52.4\%) males and 76 (46.9\%) females; one participant did not indicate a gender. The majority of participants indicated they had a Bachelor's degree or higher $(n=120 ; 74 \%)$. Only three $(2 \%)$ participants indicated that they 


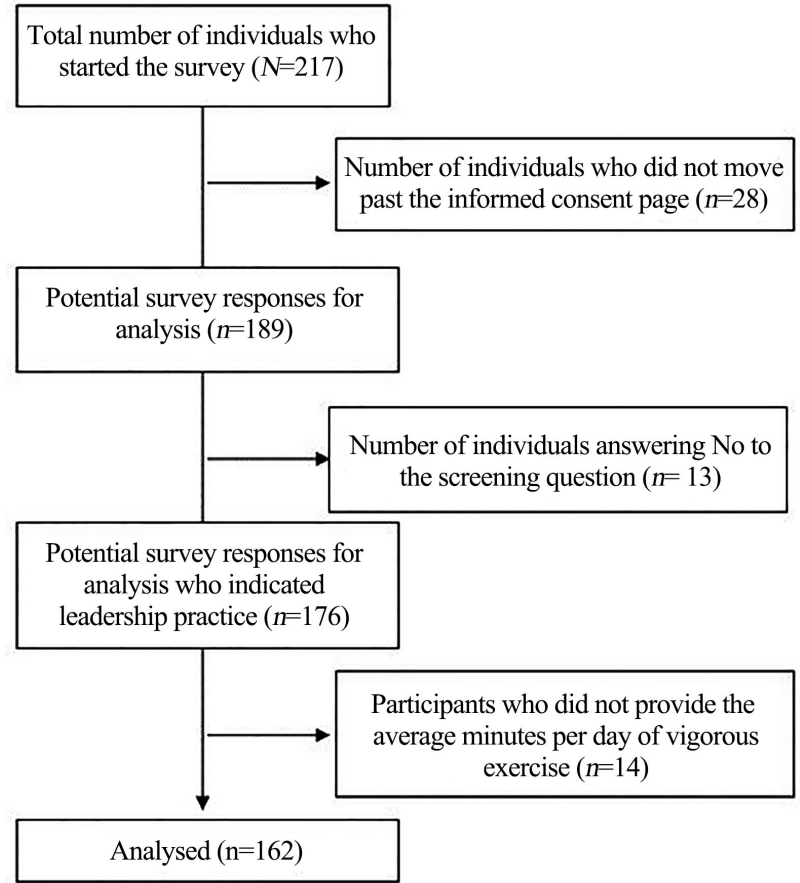

Figure 1. Participant flow chart diagram. This figure outlines the data cleaning steps performed on the responses from the online survey.

never attended college. The majority of participants indicated a household income of more than $\$ 100,000$ a year $(n=82 ; 51 \%)$. Of the 162 responses, only 12 identified their ethnicity as something other than White/Caucasian $(\mathrm{n}=150$; 93\%) (i.e., Asian ( $\mathrm{n}=2 ; 1 \%)$, Hispanic, Latino or of Spanish origin $(\mathrm{n}=3 ; 2 \%)$, Pacific Islander $(n=1,<1 \%)$, and other $(n=6 ; 4 \%))$.

\subsection{Data Collection Tools}

A digital survey was constructed and deployed to capture participant data. The following screening question was used: "Do you use or practice leadership in your work or community life (i.e., teams, groups, organizations, committees, etc.)?" Individuals who answered "no" to this question were not allowed to continue with the survey and were directed to a page thanking them for their participation. Individuals who answered "yes" to the question continued to the series of questions that collected the demographic information about each participant.

For the purpose of this study, the variable of vigorous physical activity was defined as activity that leaves participants with elevated breathing rates and sweat development after only a few minutes of activity and the inability to speak without pausing for breath, high rates of perceived exertion (RPE), and HR max elevated above 85\% of resting baseline (Mayo Clinic Staff, 2016; Norton et al., 2010, World Health Organization (WHO), n.d.). The tool selected to measure these activities was the IPAQ: Short Self-administered questionnaire (IPAQ Short Self-Administered Format, 2002). The IPAQ is designed to provide a common 
instrument that can be used to obtain internationally comparable data on health-related physical activity. The IPAQ has been subjected to numerous tests for validity and reliability (Craig et al., 2003; Hallal \& Victora, 2004). For this study, a 7-day vigorous physical activity minutes score was calculated for each participant by multiplying the number of days the participant indicated they participated in vigorous physical activity in the last week by the number of minutes the participant indicated they usually participated in the vigorous activities on one of those days in the last week (IPAQ Scoring Protocol, n.d.).

The five characteristics of servant leadership, which are altruistic calling, emotional healing, wisdom, persuasive mapping, and organizational stewardship, were measured using the self-rated Servant Leadership Questionnaire (Barbuto \& Wheeler, 2006). The Servant Leadership Questionnaire attempts to provide a reliable metric for measuring the characteristics (which included both cognitive and behavioral elements) included in the normative theory of servant leadership. For this study, the self-report version of the questionnaire was used. Data were collected by asking participants to use a 5 -point Likert scale $(1-$ not at all to 5-frequently, if not always) to respond to statements related to each of the five individual servant leadership characteristics. Each servant leadership characteristic has four to five items that make up the factor. Therefore, a mean score for each servant leadership characteristic was calculated for each participant based on the responses to each item within that factor and was used in the analysis.

\subsection{Data Analysis}

Data were scored and processed according to the data handling protocols for each of the questionnaires (i.e., the IPAQ and SLQ). Descriptive statistics (including mean, median, standard deviation, frequency, and range) were calculated for all demographic questions and for each variable. A scatter plot was created plotting the computed means of each servant leadership characteristic in relation to the indicated amount of total weekly vigorous activity. This was performed to visually demonstrate any linear relationship between the variables. Finally, bi-variate Pearson correlations were calculated to investigate the statistical relationships between the variables.

\section{Results}

In this descriptive, cross-sectional survey study of leader practitioners it was found that the mean scores for the five servant leadership characteristics were substantially higher than means scores from previous research using the same questionnaire (Cook, 2015; Barbuto \& Gifford, 2010; Barbuto \& Wheeler, 2006; Garber et al., 2009). The survey sample mirrored the demographic makeup of the local population, but reported on average participating in approximately four times more vigorous activity than the population average. In the end, no statistically significant relationship was found between the servant leadership characteristics and participation in vigorous physical activity. 


\subsection{Vigorous Physical Activity}

Table 1 presents the set of descriptive statistics for the variable vigorous physical activity. Of the 162 total responses on the survey, every respondent indicated they had participated in at least 2 days of vigorous activity in the past week (median $=5.00$ days; $\mathrm{M}=5.19$ days; $\mathrm{SD}=1.44$ ). Participants reported an average of 56.46 minutes $(\mathrm{SD}=30.86$; median $=50.00$ minutes $)$ per day of vigorous physical activity. For this participant sample, the average total vigorous physical activity per week was 301.80 minutes $(S D=190.71$; median $=275.00$ minutes). This means participants in this study on average participated in approximately 43 minutes of vigorous physical activity each day of the week.

\subsection{Servant Leadership Characteristics}

Table 2 presents the set of descriptive statistics for the variable servant leadership characteristics. On average, the frequency participants reported they used each servant leadership characteristic fell into the fairly often or frequently, if not always categories. The servant leadership characteristic participants reported using with the highest frequency was Wisdom $(\mathrm{M}=4.24$; $\mathrm{SD}=0.50)$, followed by Organizational Stewardship $(\mathrm{M}=4.05$; $\mathrm{SD}=0.57)$. The servant leadership characteristics participants reported using with the least frequency were Emotional Healing $(\mathrm{M}=3.49 ; \mathrm{SD}=0.79)$ and Persuasive Mapping $(\mathrm{M}=3.77$; $\mathrm{SD}=$ 0.63). The maximum range of the scale (i.e., a score of 5-frequently, if not always) was used by at least one participant for all servant leadership characteristics; however, the minimum range of the scale (i.e., a score of 1, not at all) was not used by at least one participant for any of the characteristics, with the lowest factor mean for any participant at 1.50 in the Emotional Healing characteristic.

Table 1. Vigorous activity descriptive statistics.

\begin{tabular}{ccccccc}
\hline & $\mathrm{N}$ & Minimum & Maximum & Mean & Median & Standard Deviation \\
\hline IPAQ Vigorous_days/week & 162 & 2 & 7 & 5.19 & 5.00 & 1.44 \\
IPAQ Vigorous_-minutes per day & 162 & 10 & 180 & 56.46 & 50.00 & 30.86 \\
Vigorous Total-days x minutes per day & 162 & 20.00 & 900.00 & 301.80 & 275.00 & 190.71 \\
\hline
\end{tabular}

Table 2. Servant leadership questionnaire descriptive statistics.

\begin{tabular}{|c|c|c|c|c|c|}
\hline & $N$ & Minimum & Maximum & Mean & $\begin{array}{c}\text { Standard } \\
\text { Deviation }\end{array}$ \\
\hline Altruistic Calling & 162 & 2.00 & 5.00 & 3.81 & 0.70 \\
\hline Emotional Healing & 162 & 1.50 & 5.00 & 3.49 & 0.79 \\
\hline Wisdom & 162 & 2.60 & 5.00 & 4.25 & 0.50 \\
\hline Persuasive Mapping & 162 & 2.20 & 5.00 & 3.77 & 0.63 \\
\hline Organizational Stewardship & 162 & 2.40 & 5.00 & 4.05 & 0.57 \\
\hline
\end{tabular}




\subsection{Cronbach's Coefficients}

Cronbach's alpha coefficient is a value between 0 and 1 that provides a measure of internal consistency and reliability of a scale (Tavakol \& Dennick, 2011). This internal consistency describes the level at which the different items in a subscale measure the same idea. Acceptable scores are greater than 0.70 and less than 0.90 (Tavakol \& Dennick, 2011). Each of the servant leadership characteristics for this study reached the 0.70 threshold. Barbuto \& Wheeler's (2006) initial study showed reliabilities of 0.77 (Altruistic Calling), 0.68 (Emotional Healing), 0.87 (Wisdom), 0.83 (Persuasive Mapping), and 0.83 (Organizational Stewardship) in their attempt to establish internal reliability of the servant leadership subscales. In comparing the alpha scores between the current study and the initial Barbuto \& Wheeler (2006) study, it is interesting to note that the two lowest subscale scores in the Barbuto and Wheeler study for Altruistic Calling and Emotional Healing were the two highest for the current study. Additional uses of the Servant Leadership questionnaire self-rater version have yielded reliable overall alphas of 0.82 (Barbuto \& Gifford, 2010), 0.90 (Cook, 2015), and 0.89 (Garber, Mardigan, Click, \& Fitzpatrick, 2009), compared to this study's overall alpha of 0.87 .

\subsection{Scatter Plot Analysis}

To visually explore the relationship between vigorous activity and each of the five servant leadership characteristics, scatter plots were created. Visually, all five scatter plots displayed the same amorphous, non-structured appearance that is depicted in the example scatter plot in Figure 2.

\subsection{Correlation of Vigorous Physical Activity and Servant Leadership Characteristics}

To further examine the relationship between self-reported weekly minutes of vigorous physical activity and servant leadership, a Pearson Correlation was performed. As seen in Table 3, no significant correlation $(r)$ was found between vigorous physical activity and any of the servant leadership characteristics. The correlations between vigorous physical activity and the servant leadership

Table 3. Correlations between servant leadership characteristics and vigorous activity.

\begin{tabular}{|c|c|c|c|c|c|}
\hline Measure & 1 & 2 & 3 & 4 & 5 \\
\hline 1) Altruistic Calling & - & & & & \\
\hline 2) Emotional Healing & $0.44^{*}$ & - & & & \\
\hline 3) Wisdom & $0.28^{*}$ & $0.17^{\star}$ & - & & \\
\hline 4) Persuasive Mapping & $0.21^{*}$ & $0.36^{*}$ & $0.31^{*}$ & - & \\
\hline 5) Organizational Stewardship & $0.34^{*}$ & $0.42^{*}$ & $0.22^{*}$ & $0.43^{*}$ & - \\
\hline 6) Vigorous Activity - min./week & 0.02 & -0.02 & 0.02 & 0.05 & 0.05 \\
\hline
\end{tabular}

*. Correlation is significant at the $p<.05$ level (2-tailed). 


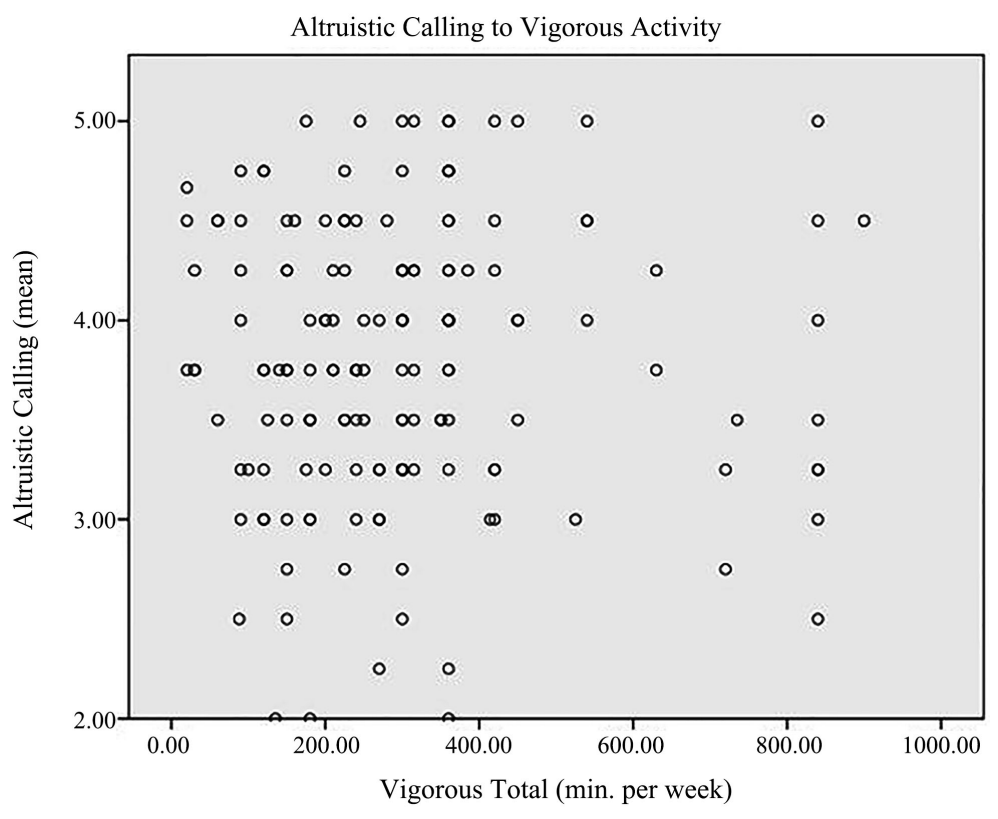

Figure 2. Scatter plot of individual altruistic calling means and vigorous activity total.

characteristics were all in the positive direction, except for Emotional Healing ( $r$ $=-0.21)$. Results of the Pearson Correlation found significant $(p<.05)$, positive correlations between all five of the servant leadership characteristics.

\section{Discussion}

The study of physical activity in the realm of leadership is still a budding research arena. Results of this study indicate that there is no statistically significant relationship between participation in vigorous physical activity and servant leadership characteristics among a sample of individuals from a southern Midwestern state sample population. From the $r$ values and interpreting the corresponding scatter plots for each relationship, we see that these two variables demonstrate a high amount of statistical independence. Two caveats regarding the sample population help contextualize the results: 1) a subset of the population that participates in the average, or less, amount of vigorous activity (75 minutes or less) and that reports low servant leadership characteristics (2.5 or less) are absent from the survey respondents; and 2) there is also a lack of participants in the extremely high amount of vigorous activity (800 minutes or more). This study design may have effectively captured high performers of both variables, thus we were unable to establish a statistical relationship between the two without having a sample of low performers.

However, we also know that vigorous physical activity has an established benefit in a wide range of personal health outcomes (Karlsen et al., 2017). The conscious and purposeful choice of vigorous physical activity has been shown to allow people to reap physical benefits and performance benefits for individuals who want to be able to tolerate and perform in a broader range of activity levels 
across wider time ranges (Ekkekakis, Parfitt, \& Petruzzello, 2012; Jung, Bourne, \& Little, 2014). It has also been observed that the conscious choice of servant leadership not only accomplishes organizational goals, but also is able to promote and foster the growth of leader's subordinates (Ng, Koh, \& Goh, 2008). Conceptually, vigorous physical activity and servant leadership seem to have in common some of the same required cognitive characteristics that would be needed to carry out these behaviors, such as inner-drive, discipline, and the ability to step out of one's comfort zone (Kaus, 2014; Neubert et al., 2013). However, based on the results of this study, it may be the case that, no matter the level of vigorous physical activity participation an individual pursues, they may or may not use servant leadership characteristics in their leadership practice. And vice versa, the frequency of the use of servant leadership characteristics may not be related to the amount of vigorous physical activity in which an individual participates.

Individuals who put the concerns of followers first (i.e., who practice servant leadership) can be people who participate in very little vigorous physical activity and can be people who participate in a lot of vigorous physical activity. Also, individuals who participate in a lot of vigorous physical activity can be people who put the concerns of followers first and can be people who use a different philosophy of leadership (i.e., transformative, leader-member exchange, authentic, transactional, etc.). This study found that while participation in vigorous physical activity and usage of servant leadership characteristics may not be empirically linked, more research is still needed to explore some of the underlying motivations and influences of these variables.

Future research could begin by exploring participants' beliefs about the benefits of practicing and participating in each of the variables to determine the driving motivations that influence each behavior. For example, in light of these empirical findings, it may be beneficial to build off the qualitative work of Himelhoch (2014) to further explore the contextually and situationally grounded motivational ties between high-intensity exercise participation and leadership practice. While Himelhoch's (2014) research focused on transformational leadership with a small sample size $(\mathrm{N}=5)$ of established upper-level managers and company presidents, these leaders indicated strong personal ties between their training and leadership practices. It is not possible, based on the current study, to examine the motivations driving activity participation and leadership practice; only the level of each of the variables was measured and it does not appear to influence the level of the other in the abstract sense. Although no definitive solution to understanding the relationship between vigorous physical activity and servant leadership was available based on this one study, one can still establish reasonable boundaries around recommendations for the basic application of these findings to pratice.

An issue that has arisen in the United States is the rise of fitness centers as profitable businesses with a focus on weight loss as a driver for physique and aesthetics instead of health and wellness ("Schools, Families, and Communities," 
n.d.). If the primary focus is on appearance, it sets up a large amount of failure in gym attendance and program adherence (Mustain, 2014). These types of goals contain weak personal extrinsic motivations and lack strong intrinsic motivations that would have lasting effects (Edmunds, Ntoumanis, \& Duda, 2007). By getting fitness professionals, coaches, and trainers to shift their focus in promoting the benefits of physical activity from aesthetics to measurable health and wellness improvements, the individual could be holistically developed. The growth of functional fitness (i.e., CrossFit, Orange Theory, etc.) provides a promising step as larger populations are being exposed to varying intensity levels and general health recommendations. These burgeoning programs place a firm priority on overall health improvement, encourage their clientele to take ownership of their fitness, and present a social environment for their physical activity practice in group settings.

Growing the size of the population that participates in the recommended amount of physical activity is dependent on creating environments where it is easy to participate in regular, health-beneficial physical activity. The greatest influencers in these environments are the respective community and organizational leaders. In some ways, it was fortunate that this study did not find a statistical relationship between vigorous activity participation and servant leadership characteristics, because the absence of a relationship suggests that the exclusive use of the servant leadership philosophy to promote vigorous physical activity participation is not needed. The findings demonstrate that the level of vigorous physical activity participation does not seem to matter when it comes to servant leadership practice. This is good news as it does not limit practicing leaders, with varying leadership philosophies, from the promotion of physical activity.

All leaders have the potential to facilitate physical activity in personal and workplace environments. The utilization of situational leadership through vision and foresight could be useful to promote physical activity participation for all workplace environments (Hershey \& Blanchard, 1969). Collectively, there are many ways to promote and motivations for physical activity to be further incorporated into the personal and professional lives of today's leaders and followers. The conscious choice of leaders to instill and demonstrate these characteristics personally and professionally can serve as the best practice in organizational and personnel health and wellness. The apparent independence of the studied variables, as seen from the lack of correlation, demonstrates how the motivating factors are not exclusively demonstrated by vigorous activity participants and practitioners of servant leadership.

Organizational leaders have a responsibility to promote healthy environments, including physical activity participation and positive leadership practices. The demonstrated lack of a statistical relationship between the variables opens the door for any practicing leader to encourage physical activity participation and revise appropriate polices related to the creation of environments that make physical activity participation easier. Community leaders also have a responsibil- 
ity, through positive leadership practices, to remove barriers and create opportunities for all members of the community to have access to viable physical activity outlets. Bike lanes, parks, walking trails, and community recreational activities need to be maintained, promoted, and valued as outlets for physical activity (Heath et al., 2006; Tuso, 2015). The placement of these health-promoting design elements is not only a positive leadership practice, in the theme of servant leadership, but also serves to promote physical activity participation for the full demographic spectrum regardless of location or socioeconomic standing.

\section{Conclusion}

With the very real and growing problem of the rising rates of metabolic syndrome, and its effects on health, wellness, performance, and engagement, an effective method for its combat are necessary. Physical activity and healthy lifestyle choices are the most effective and lasting influence on this set of chronic diseases. Even with the known information, physical activity levels across the country are persistently under the recommended levels. One known effective way to promote physical activity is to create work cultures which encourage healthy lifestyles and physical activity supported through altruistic and participatory leadership. Physical activity and the application of practical leadership theories provide an option to facilitate such an environment.

An attempt was made to examine the relationship between two variables in a local population, vigorous activity participation measured by the IPAQ and servant leadership characteristics measured by the SLQ. While a significant relationship was not found, high levels of each variable were discovered independently in the sample population. Using collected demographic information, it was possible to detail characteristics of high level practitioners of both variables. From this data, it was proposed that health care, fitness industry, and community/organizational leadership practitioners should evolve their promotion of physical activity and purposefully spread its usage across more diverse age, ethnic, and socioeconomic groups. The increased usage of physical activity has the potential to ward off the growing prevalence of metabolic syndrome before a larger scale epidemic occurs which could further compromise the performance and engagement of the United States workforce (King et al., 1988).

The absence of evidence to support the conclusion that a relationship exists between the two variables allows for a large number of methods, styles, and theories to be used in the promotion of physical activity. The promotion of environments that foster physical activity and healthy lifestyles across all demographics in the fitness industry, healthcare, and organizational and community leaders provides the best solution to the growing rates of metabolic syndrome.

\section{Conflicts of Interest}

The authors declare no conflicts of interest regarding the publication of this paper. 


\section{References}

Ahlskog, J. E. (2011). Does Vigorous Exercise Have a Neuroprotective Effect in Parkinson Disease? Neurology, 77, 288-294. https://doi.org/10.1212/WNL.0b013e318225ab66

Bailey, R. (2006). Physical Education and Sport in Schools: A Review of Benefits and Outcomes. Journal of School Health, 76, 397-401. https://doi.org/10.1111/j.1746-1561.2006.00132.x

Barbuto, J. E., \& Gifford, G. T. (2010). Examining Gender Differences of Servant Leadership: An Analysis of the Agentic and Communal Properties of the Servant Leadership Questionnaire. Journal of Leadership Education, 9, 4-23.

https://doi.org/10.12806/V9/I2/RF1

https://journalofleadershiped.org/vol-9-iss-2/158-examining-gender-diffe-rences-of-se rvant-leadership-an-analysis-of-the-agentic-and-communal-properties-of-servant-lead ership-questionnaire

Barbuto, J. E., \& Wheeler, D. W. (2006). Scale Development and Construct Clarification of Servant Leadership. Group \& Organization Management, 31, 300-326. https://doi.org/10.1177/1059601106287091

Beachamp, M. R., Welch, A. S., \& Hulley, A. J. (2007). Transformational and Transactional Leadership and Exercise-Related Self-Efficacy: An Exploratory Study. Journal of Health Psychology, 12, 83-88. https://doi.org/10.1177/1359105307071742

Boles, M., Pelletier, B., \& Lynch, W. (2004). The Relationship between Health Risks and Work Productivity. Journal of Occupational \& Environmental Medicine, 46, 737-745. https://doi.org/10.1097/01.jom.0000131830.45744.97 https://journals.lww.com/joem/Abstract/2004/07000/The_Relationship_Between_Healt h_Risks_and_Work.18.aspx

Centers for Disease Control and Prevention (2013). Adult Participation in Aerobic and Muscle-Strengthening Physical Activities-United States, 2011. Morbidity and Mortality Weekly Report, 62, 326-330.

https://www.cdc.gov/mmwr/preview/mmwrhtml/mm6217a2.htm?s_cid=mm6217a2_w

Collingwood, T. R. (1997). Providing Physical Fitness Programs to at-Risk Youth. Quest, 49, 67-84. https://doi.org/10.1080/00336297.1997.10484224

Cook, K. F. (2015). Self-Perceived Traits of Servant Leadership in AmeriCorps Volunteers: A Mixed Method Concurrent Explanatory Study. Creighton Journal of Interdisciplinary Leadership, 1, 24-50. https://doi.org/10.17062/cjil.v1i1.21 https://dspace2.creighton.edu/xmlui/bitstream/handle/10504/70819/21-141-2-PB.pdf?s equence $=1$ \&isAllowed $=y$

Craig, C. L., Marshall, A. L., Sjostrom, M., Bauman, A. E., Booth, M. L., Ainsworth, B. E., Ekelund, U. et al. (2003). International Physical Activity Questionnaire: 12-Country Reliability and Validity. Medicine and Science in Sports and Exercise, 35, 1381-1395. https://doi.org/10.1249/01.MSS.0000078924.61453.FB

Edmunds, J., Ntoumanis, N., \& Duda, J. L. (2007). Testing a Self-Determination TheoryBased Teaching Style Intervention in the Exercise Domain. European Journal of Social Psychology, 38, 375-388. https://doi.org/10.1002/ejsp.463

Ekkekakis, P., Parfitt, G., \& Petruzzello, S. J. (2012). The Pleasure and Displeasure People Feel When They Exercise at Different Intensities. Sports Medicine, 41, 641-671. https://doi.org/10.2165/11590680-000000000-00000

Ferron, C., Narring, F., Cauderay, M., \& Michaud, P. A. (1999). Sport Activity in Adolescence: Associations with Health Perceptions and Experimental Behaviours. Health Education Research, 14, 225-233. https://doi.org/10.1093/her/14.2.225

Garber, J. S., Mardigan, E. A., Click, E. R., \& Fitzpatrick, J. J. (2009). Attitudes towards 
Collaboration and Servant Leadership among Nurses, Physicians and Residents. Journal of Interprofessional Care, 23, 331-340. https://doi.org/10.1080/13561820902886253

Gebel, K., Ding, D., Chey, T., Stamatikis, E., Brown, W. J., \& Baumann, A. E. (2015). Effect of Moderate to Vigorous Physical Activity on All-Cause Mortality in Middle-Aged and Older Australians. JAMA Internal Medicine, 175, 970-977. https://doi.org/10.1001/jamainternmed.2015.0541

Greenleaf, R. K. (1977). Servant Leadership: A Journey into the Nature of Legitimate Power and Greatness. Mahwah, NJ: Paulist Press.

Gutin, B., \& Kasper, M. J. (1992). Can Vigorous Exercise Play a Role in Osteoporosis Prevention? A Review. Osteoporosis International, 2, 55-69. https://doi.org/10.1007/BF01623838

Hallal, P. C., \& Victora, C. G. (2004). Reliability and Validity of the International Physical Activity Questionnaire (IPAQ). Medicine and Science in Sports and Exercise, 36, 556. https://doi.org/10.1249/01.MSS.0000117161.66394.07

Heath, G. W., Brownson, R. C., Kruger, J., Miles, R., Powell, K. E., \& Ramsey, L. T. (2006). The Effectiveness of Urban Design and Land Use and Transport Policies and Practices to Increase Physical Activity: A Systematic Review. Journal of Physical Activity and Health, 3, 55-76. https://doi.org/10.1123/jpah.3.s1.s55

Hershey, P., \& Blanchard, K. H. (1969). Management of Organizational Behavior. Academy of Management Journal, 12, 526.

https://search.proquest.com/openview/6b62a3c08c14c82dde2a4b50949e40b8/1?pq-orig site $=$ gscholar

Himelhoch, C. R. (2014). Transformational Leadership: And High-Intensity Interval Training (Vol. 1). Spring Lake, MI: MindBodyMed Press.

Hupin, D., Roche, F., Gremeaux, V., Chatard, J. C., Oriol, M., Gaspoz, J. M., \& Edouard, P. (2015). Even a Low-Dose of Moderate-to-Vigorous Physical Activity Reduces Mortality by $22 \%$ in Adults Aged $\geq 60$ Years: A Systematic Review and Meta-Analysis. Journal of Sports Medicine, 49, 1262-1267.

https://doi.org/10.1136/bjsports-2014-094306

IPAQ Scoring Protocol.

https://sites.google.com/site/theipaq/scoring-protocol

IPAQ Short Self-Administered Format (Questionnaire) (2002). Unpublished Instrument. https://sites.google.com/site/theipaq/questionnaire_links

Jung, M. E., Bourne, J. E., \& Little, J. P. (2014). Where Does HIT Fit? An Examination of the Affective Response to High-Intensity Intervals in Comparison to Continuous Moderate- and Continuous Vigorous-Intensity Exercise in the Exercise Intensity-Affect Continuum. PLoS ONE, 9, e114541. https://doi.org/10.1371/journal.pone.0114541

Karlsen, T., Aamot, I. L., Haykowsky, M., \& Rognmo, O. (2017). High Intensity Interval Training for Maximizing Health Outcomes. Progress in Cardiovascular Disease, 60, 67-77. https://doi.org/10.1016/j.pcad.2017.03.006

Kaus, R. J. (2014). Affect and Enjoyment Associated with CrossFit Exercise. Master's Thesis, Bowling Green, OH: Bowling Green State University. https://etd.ohiolink.edu/rws_etd/document/get/bgsu1395427844/inline

Keith, K. (2013). Servant Leadership. In W. G. Rowe, \& L. Guerrero (Eds.), Cases in Leadership. Thousand Oaks, CA: Sage Publications.

King, A. C., Carl, F., Birkel, L., \& Haskell, W. L. (1988). Increasing Exercise among Blue-Collar Employees: The Tailoring of Worksite Programs to Meet Specific Needs. Preventative Medicine, 17, 357-365. 
Kravitz, L. (2014). Metabolic Effects of HIIT. IDEA Fitness Journal, 11, 16-18. http://www.unm.edu/ lkravitz/Article\%20folder/metabolicEffectsHIIT.html

Kwoh, L. (2013). Want to Be CEO? What's Your BMI? The Wall Street Journal. https://www.wsj.com/articles/SB10001424127887324595704578241573341483946

Mayo Clinic Staff (2016). Exercise Intensity: How to Measure It. https://www.mayoclinic.org/healthy-lifestyle/fitness/in-depth/exercise-intensity/art-20 046887

McDowell-Larsen, S., Kearney, L., \& Campbell, D. (2002). Fitness and Leadership: Is There a Relationship?: Regular Exercise Correlates with Higher Leadership Ratings in Senior-Level Executives. Journal of Managerial Psychology, 14, 316-324. https://doi.org/10.1108/02683940210428119

Morgan, W. P. (1985). Affective Beneficence of Vigorous Physical Activity. Medicine \& Science in Sports \& Exercise, 17, 94-100. https://doi.org/10.1249/00005768-198502000-00015

Mustain, P. (2014). What If We All Just Stopped Trying to Lose Weight? Scientific American.

https://blogs.scientificamerican.com/food-matters/what-if-we-all-just-stopped-trying-t o-lose-weight-video/

Neubert, M. J., Penney, L. M., Perry, S. J., Weinberger, E., Witt, L. A., \& Hunter, E. M. (2013). Servant Leaders Inspire Servant Followers: Antecedents and Outcomes for Employees and the Organization. The Leadership Quarterly, 24, 316-331. https://doi.org/10.1016/j.leaqua.2012.12.001

Ng, K., Koh, C. S., \& Goh, H. (2008). The Heart of the Servant Leader: Leader's Motivation-to-Serve and Its Impact on LMX and Subordinates' Extra-Role Behaviors. In Knowledge-Driven Corporation: Complex Creative Destruction (pp. 125-144). https://doi.org/10.1037/e518532013-560 https://books.google.com/

Northouse, P. G. (2012). Leadership: Theory and Practice. Thousand Oaks, CA: Sage Publications.

Norton, K., Norton, L., \& Sadgrove, D. (2010). Position Statement on Physical Activity and Exercise Intensity Terminology. Journal of Science and Medicine in Sport, 13, 496-502. https://doi.org/10.1016/j.jsams.2009.09.008

Ozanian, M. (2015). How CrossFit Became a \$4 Billion Brand. Forbes. https://www.forbes.com/sites/mikeozanian/2015/02/25/how-crossfit-became-a-4-billio n-brand/\#810eeab78c17

Robson, S. (2013). Physical Fitness and Resilience: A Review of Relevant Constructs, Measures, and Links to Well-Being. Santa Monica, CA: Rand Corporation. https://www.rand.org/content/dam/rand/pubs/research_reports/RR100/RR104/RAND RR104.pdf

Schools, Families, and Communities. http://www.uconnruddcenter.org/schools-families-communities

Swain, D. P., \& Franklin, B. A. (2006). Comparison of Cardioprotective Benefits of Vigorous versus Moderate Intensity Aerobic Exercise. The American Journal of Cardiology, 97, 141-147. https://doi.org/10.1016/j.amjcard.2005.07.130

Tavakol, M., \& Dennick, R. (2011). Making Sense of Cronbach's Alpha. International Journal of Medical Education, 2, 53-55. https://doi.org/10.5116/ijme.4dfb.8dfd

Taylor, C. B., Sallis, J. F., \& Needle, R. (1985). The Relation of Physical Activity and Exercise to Mental Health. Public Health Reports, 100, 195-202. 
https://www.ncbi.nlm.nih.gov/pmc/articles/PMC1424736/

Thayer, D., Konicki, D. L., Yarborough, C. M., Peterson, K. W., Issac, F., Loppke, R., Fabius, R. et al. (2013). The Link between Workforce Health and Safety and the Health of the Bottom Line: Tracking Market Performance of Companies that Nurture a "Culture of Health". Journal of Occupational Environmental Medicine, 55, 993-1000. https://doi.org/10.1097/JOM.0b013e3182a6bb75

Tudor-Locke, C., Johnson, W., \& Katzmarzyk, P. T. (2010). Frequently Reported Activities by Intensity for U.S. Adults: The American Time Use Survey. American Journal of Preventive Medicine, 39, e13-e20. https://doi.org/10.1016/j.amepre.2010.05.017

Tuso, P. (2015). Strategies to Increase Physical Activity. The Permanente Journal, 19, 84-88. https://doi.org/10.7812/TPP/14-242

Wankel, L. M., \& Berger, B. G. (2018). The Psychological and Social Benefits of Sport and Physical Activity. Journal of Leisure Research, 22, 167-182. https://doi.org/10.1080/00222216.1990.11969823

Williams, P. T. (2004). Vigorous Exercise and the Population Distribution of Body Weight. International Journal of Obesity, 28, 120-128. https://doi.org/10.1038/sj.ijo.0802480

World Health Organization. What Is Moderate-Intensity and Vigorous-Intensity Physical Activity? https://www.who.int/dietphysicalactivity/physical_activity_intensity/en/

Wrynn, A. (2003). Contesting the Canon: Understanding the History of the Evolving Discipline of Kinesiology. Quest, 55, 244-256. https://doi.org/10.1080/00336297.2003.10491802

Youngstedt, S. D. (2005). Effects of Exercise on Sleep. Clinics in Sports Medicine, 24, 355-365. https://doi.org/10.1016/j.csm.2004.12.003 